\title{
Gene-based SNP discovery and genetic mapping in pea
}

\author{
Anoop Sindhu $\cdot$ Larissa Ramsay $\cdot$ Lacey-Anne Sanderson $\cdot$ Robert Stonehouse $\cdot$ Rong Li $\cdot$ Janet Condie $\cdot$ \\ Arun S. K. Shunmugam · Yong Liu • Ambuj B. Jha · Marwan Diapari · Judith Burstin · Gregoire Aubert • \\ Bunyamin Tar'an · Kirstin E. Bett · Thomas D. Warkentin · Andrew G. Sharpe
}

Received: 15 February 2014 / Accepted: 29 July 2014 / Published online: 15 August 2014

(C) The Author(s) 2014. This article is published with open access at Springerlink.com

\begin{abstract}
Key message Gene-based SNPs were identified and mapped in pea using five recombinant inbred line populations segregating for traits of agronomic importance.

Abstract Pea (Pisum sativum L.) is one of the world's oldest domesticated crops and has been a model system in plant biology and genetics since the work of Gregor Mendel. Pea is the second most widely grown pulse crop in the world following common bean. The importance of pea as a food crop is growing due to its combination of moderate protein concentration, slowly digestible starch, high
\end{abstract}

Communicated by Antonio Augusto Franco Garcia.

Electronic supplementary material The online version of this article (doi:10.1007/s00122-014-2375-y) contains supplementary material, which is available to authorized users.

A. Sindhu $\cdot$ L.-A. Sanderson $\cdot$ R. Stonehouse $\cdot$

A. S. K. Shunmugam · Y. Liu - A. B. Jha - M. Diapari · B. Tar'an ·

K. E. Bett · T. D. Warkentin $(\square)$

Department of Plant Sciences, Crop Development Centre,

University of Saskatchewan, 51 Campus Drive, Saskatoon,

SK S7N 5A8, Canada

e-mail: tom.warkentin@usask.ca

L. Ramsay · R. Li · J. Condie · A. G. Sharpe $(\bowtie)$

National Research Council Canada, 110 Gymnasium Place,

Saskatoon, SK S7N 0W9, Canada

e-mail: andrew.sharpe@nrc-cnrc.gc.ca

Present Address:

L. Ramsay

Department of Plant Sciences, Crop Development Centre,

University of Saskatchewan, 51 Campus Drive, Saskatoon,

SK S7N 5A8, Canada

J. Burstin · G. Aubert

UMR1347 Agroecology, INRA, 17 rue de Sully,

21065 Dijon Cedex, France dietary fiber concentration, and its richness in micronutrients; however, pea has lagged behind other major crops in harnessing recent advances in molecular biology, genomics and bioinformatics, partly due to its large genome size with a large proportion of repetitive sequence, and to the relatively limited investment in research in this crop globally. The objective of this research was the development of a genome-wide transcriptome-based pea single-nucleotide polymorphism (SNP) marker platform using next-generation sequencing technology. A total of 1,536 polymorphic SNP loci selected from over 20,000 non-redundant SNPs identified using deep transcriptome sequencing of eight diverse Pisum accessions were used for genotyping in five RIL populations using an Illumina GoldenGate assay. The first high-density pea SNP map defining all seven linkage groups was generated by integrating with previously published anchor markers. Syntenic relationships of this map with the model legume Medicago truncatula and lentil (Lens culinaris Medik.) maps were established. The genic SNP map establishes a foundation for future molecular breeding efforts by enabling both the identification and tracking of introgression of genomic regions harbouring QTLs related to agronomic and seed quality traits.

\section{Introduction}

Pea (Pisum sativum L., $2 n=2 x=14$ ) belongs to the Fabaceae family of flowering plants which includes other important agricultural crops such as soybean, chickpea, lentil, alfalfa, peanut, and common bean. Being a rich source of protein, polysaccharides and providing slowly digestible starch, soluble sugars, fiber, minerals, and vitamins, pea plays an important role in meeting human nutritional needs (Smýkal et al. 2012). In addition to the use of 
mature seeds for food and feed, the pea crop can be used as a vegetable, forage, silage, or green manure. Like other legumes, pea contributes to the development of low-input farming systems by fixing atmospheric nitrogen, and serves as a break crop which reduces the need for pesticide inputs. Global dry pea cultivation over the past decade has ranged from 6.0 to 6.5 million hectares producing 9.4-11.7 million tons per year (FAOSTAT 2013). In addition, global cultivation of vegetable pea has ranged from 1.6 to 2.2 million hectares producing 12.0-17.4 million tons per year over the past decade (FAOSTAT 2013).

Genetic studies in pea have a long history, starting from the early 1790s (Thomas Andrew Knight, as indicated in Ellis 2009) to the very birth of the principles of genetics (Mendel 1865). Yet, despite being an important crop for global food security, pea has not received as much attention as other crops and has lagged behind in the development of molecular biology, genomics and bioinformatics tools (Bhattacharyya et al. 1990; Hofer et al. 2009). Along with the high cost of developing genomic resources, its large genome size ( 4 Gbp; Arumuganathan and Earle 1991), recalcitrance to transformation (Clemow et al. 2011), and lack of a reference genome sequence have been the major contributing factors hindering the development of genomic resources in pea. Thus, despite the early lead, pea has lagged behind other major crops (including wheat, rice, corn, and soybean) in terms of genomic resource development.

Developing a genome-wide arrayed class of molecular markers and generating saturated molecular maps are among the first steps in the application of molecular breeding, genomic selection, tagging and gene introgression strategies (Timmerman-Vaughan et al. 2004; Burstin et al. 2007; Lejeune-Hénaut et al. 2008). Despite being a crop species with a large genome, pea is one of the few crop species where early genetic maps were developed using phenotypic and physiological characteristics (Blixt 1974), isozymes (Weeden et al. 1998), and early DNA-based markers (Weeden et al. 1998; Ellis et al. 1992). A pea linkage map consisting of 209 markers spanning $1330 \mathrm{cM}$ was developed using AFLP, RFLP, and RAPD markers (Gilpin et al. 1997), and a consensus map derived from several segregating populations defining seven linkage groups using a combination of morphological, biochemical, and molecular markers was developed by Weeden et al. (1998).

Most of the existing markers are non-targeted (anonymous) genomic markers useful in providing a framework for genetic map development and in assessing genetic diversity, germplasm characterization and have also been used in mapping of quantitative trait loci (QTLs). However, since they are usually not part of the expressed portion of the genome, they are likely to provide, at most, indirect information on functional genes (van Treuren and van Hintum 2009). Therefore, attempts have been made to generate genic molecular markers in pea such as EST-PCR based (Ellis and Poyser 2002; Miesel et al. 2005; Aubert et al. 2006; Bordat et al. 2011), EST-derived SSR (Burstin et al. 2001; Smýkal et al. 2008; Gong et al. 2010; De Caire et al. 2011), and intron targeted markers (Brauner et al. 2002) for the construction of genetic maps. Since transcriptomic markers are derived from gene sequences, they are also useful for establishing syntenic relationships with related crop species.

With the advent of high-throughput genotyping technologies, single-nucleotide polymorphisms (SNPs) have become the marker of choice due to their abundance and the availability of high-throughput screening techniques. In humans, their frequency is about one SNP in every $1000 \mathrm{bp}$ while in soybean on average one SNP occurs in every 200-300 bp, with intronic regions showing three times higher frequency than exonic regions (Wang et al. 1998; Zhu et al. 2003). Development of several platforms for detecting SNPs has led to the refinement of the technology and now genomewide SNP databases can be generated at reduced cost and in a much shorter time compared to 10 years ago. Many crop species now have high-density SNP maps available which have proven to be valuable resources for practical applications in gene mapping, association mapping, and genomic selection (Jing et al. 2007; Vignal et al. 2002; Varshney et al. 2005). Until recently in pea, however, only limited SNP resources have been available (Aubert et al. 2006; Deulvot et al. 2010). SNP discovery and mapping carried out by Bordat et al. (2011), using 214 EST-derived markers, resulted in a resource to deploy in silico mapping of 5,460 pea unigenes using genomic resources developed in the model legume Medicago truncatula. More recently, it has been possible to generate high-throughput de novo transcriptome sequence data in pea using next-generation sequencing (NGS) technology (Franssen et al. 2011; Kaur et al. 2012). The transcriptome sequencing approach provides transcript sequences that are much shorter in length than the ESTs generated using Sanger sequencing, however, these deep data sets serve as a good resource for gene expression analysis, metabolic pathway and cellular process analysis as well as the detection and mining of genic SNPs, as has been described recently in pea (Leonforte et al. 2013; Duarte et al. 2014), as well as in other pulse crops such as lentil (Sharpe et al. 2013). Leonforte et al. (2013) described a set of 408 gene-based SNPs identified between two pea genotypes which were placed in an SSR-anchored genetic map that enabled comparative analysis with model legume genomes as well as the identification of QTLs for salt tolerance.

The objective of this research was the development of a transcriptome-based SNP marker resource in pea. We used targeted $3^{\prime}$ end cDNA sequencing of eight Pisum accessions for SNP discovery and developed a dense consensus genetic map by genotyping five diverse recombinant inbred line (RIL) populations. 


\section{Methods}

\section{Plant material}

Eight diverse pea accessions were utilized in this research including six Pisum sativum cultivars (CDC Bronco, Alfetta, Cooper, CDC Striker, Nitouche, and Orb) and two wild accessions P651 (P. fulvum), PI 358610 (P. sativum ssp. abyssinicum) (Table 1). The cultivars were developed in Canada or Western Europe and all are adapted to North American production, while the P. sativum subspp. abyssinicum and $P$. fulvum accessions were sourced from germplasm collections in USA and Spain, respectively (Table 1). The $P$. sativum subspp. abyssinicum accession (PI 358610) is a land race collected in Ethiopia (http://www.ars-grin. gov/cgi-bin/npgs/html/taxon.pl?28663). CDC Bronco (Warkentin et al. 2005) was used as the reference genome relative to which SNPs were called as it is a widely grown cultivar in Canada and is one of the parents of several RIL populations developed at the University of Saskatchewan. Line 1-2347-144, a parent in RIL population PR-15, is derived from mutagenesis of CDC Bronco, and retains the majority of the CDC Bronco traits (Warkentin et al. 2012) (Table 2). Nitouche and PI 358610 (P. sativum ssp. abyssinicum) were not parents of any of the RIL populations but were included in this research to increase genetic diversity. A single plant of each genotype was selfed to produce enough seed for tissue collection and library preparation. Leaf, stem, flowers and developing seeds were collected from plant materials grown in a growth chamber with $16 \mathrm{~h}$ light $/ 8 \mathrm{~h}$ dark with day temperature $23{ }^{\circ} \mathrm{C}$ and night temperature $15{ }^{\circ} \mathrm{C}$. Additional samples were collected from etiolated seedlings grown on filter paper soaked with sterile water in sealed Petri plates with no light. Five RIL populations, derived from diverse parents, were used for genetic mapping. All except Pop9 are from the University of Saskatchewan field pea breeding program and were developed for the purpose of mapping QTLs for traits of importance including disease resistance and nutritional value. These populations were developed by selfing using the single seed descent method from the $F_{2}$ generation, then seeds from single $\mathrm{F}_{7}$ plants were bulked. Summarized RIL population parentage and their origins are provided in Supplementary Table S1. In total 586 RILs from five populations were used for mapping. DNA was extracted using a modified CTAB method (Doyle and Doyle 1990) from freeze-dried leaf tissue collected from at least five plants of each RIL to try to assess residual heterozygosity within each RIL.

\section{$3^{\prime}$ anchored cDNA library construction and sequencing}

Five kinds of tissue samples were collected from each accession including leaf ( 2 week old), stem (before flowering), etiolated seedlings ( 1 week old), flower (mixed stages) and developing seeds (mixed stages). RNA extraction, $3^{\prime}$-cDNA library construction, and 454 Roche Titanium sequencing were carried out exactly as described in Sharpe et al. (2013). Briefly, total RNA from leaves was extracted using the RNeasy Plant Mini Kit (Qiagen) including on-column DNase digestion. Total RNA from other tissues was extracted using the CTAB method described by Miesel et al. (2005) and then cleaned up using RNeasy Mini kits (Qiagen), including on-column DNase digestion. 3'-anchored cDNA libraries for 454 sequencing were prepared based on a protocol described in Eveland et al. (2008) and modified to incorporate AciI as the restriction enzyme used to generate $3^{\prime}$ cDNA fragments of the optimal size range for amplification during 454 Titanium chemistry sequencing (Parkin et al. 2010). The Acil-digested cDNA was treated with Agencourt AMPure Beads (Beckman Coulter Inc.) to remove smaller fragments $(<250 \mathrm{bp})$. The $3^{\prime}$-fragments of cDNA were recovered using DynBeads M-270 streptavidin (Invitrogen) and then ligated with A-adaptor. Roche 454 Titanium sequencing of the titrated single strand DNA libraries was carried out following the procedure described by Margulies et al. (2005) with modifications for the Titanium chemistry as described in protocols supplied by the manufacturer (Roche, Laval, Quebec).

Sequence assembly, analysis and SSR analysis

A de novo assembly of the CDC Bronco 454 reads was performed using NGen (DNAStar) software as described in Sharpe et al. (2013) with a few differences as follows. Parameters used for the de novo assembly included: Min/ Match Percent $=90$; Max 454 Sequence Length $=600$; Repeat Handling On; and Expected Coverage $=20$. Processing was carried out on a Dell R910, $2 \times 2.40 \mathrm{GHz}$, 48 GB RAM Windows 64 Bit server. Following assembly, repeat class contigs were removed as well as any remaining contigs of $<200 \mathrm{bp}$. Sequence data from the other accessions were then assembled against the CDC Bronco de novo reference assembly using NGen (DNAStar). To ensure removal of the 454 key sequence from reads, $10 \mathrm{bp}$ were trimmed from the $5^{\prime}$ ends. Adapter screening was used to remove the wobble primer, the adapter, and any poly-A tail. Contaminant filtering was implemented using a set of four mitochondrial, chloroplast, and ribosomal sequences.

The identification of candidate SSRs in the reference assembly together with numbers of potentially polymorphic SSR loci amongst the $P$. sativum and $P$. fulvum accessions was carried out using the software QDD (Meglécz et al. 2010). The primer pairs flanking these loci were also generated using QDD. 


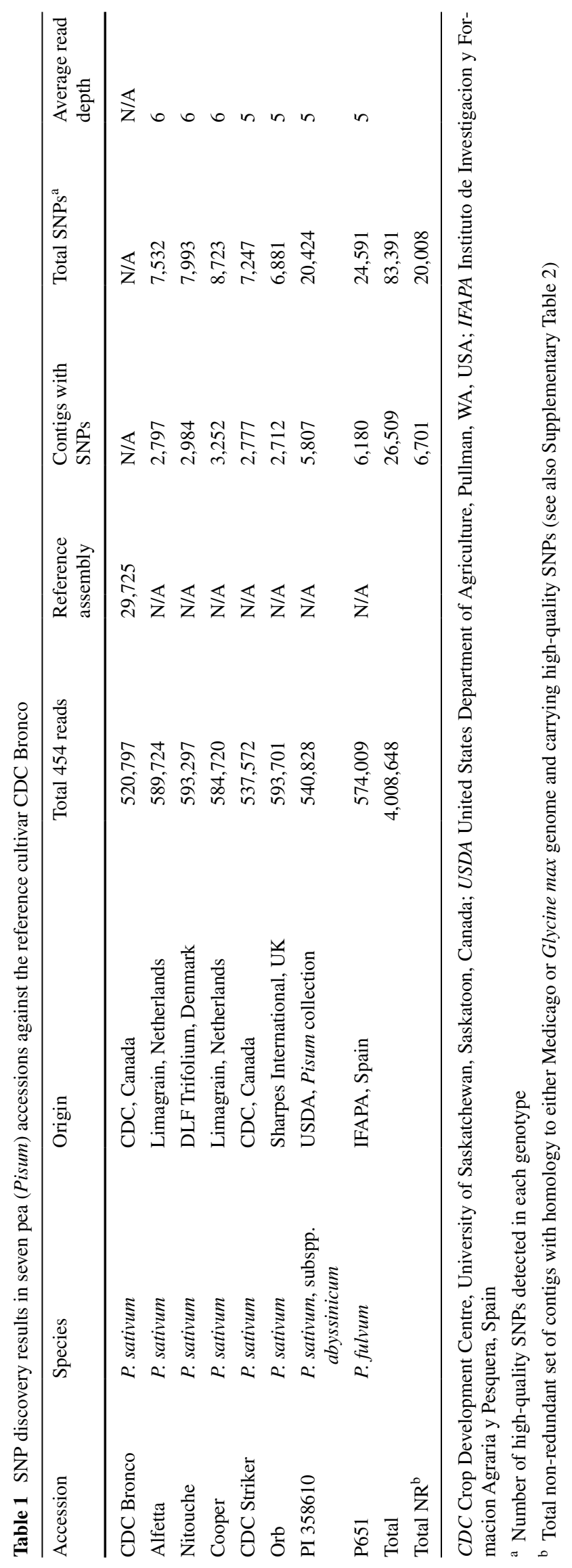


Table 2 RIL populations, SNP polymorphism, mapped markers and markers shared by each population in consensus map

\begin{tabular}{|c|c|c|c|c|c|c|c|c|c|c|}
\hline \multirow[t]{2}{*}{ Population } & \multirow[t]{2}{*}{$\begin{array}{l}\text { RIL } \\
\text { size }\end{array}$} & \multirow[t]{2}{*}{$\begin{array}{l}\text { Poly-morphic } \\
\text { SNPs }\end{array}$} & \multirow[t]{2}{*}{$\begin{array}{l}\text { Mono- } \\
\text { morphic }\end{array}$} & \multirow[t]{2}{*}{ Dominant } & \multirow[t]{2}{*}{$\begin{array}{l}\text { Failed/ } \\
\text { unscorable }\end{array}$} & \multirow[t]{2}{*}{$\begin{array}{l}\text { Mapped SNPs } \\
\text { (with SSR }{ }^{\mathrm{a}} \text { ) }\end{array}$} & \multicolumn{4}{|c|}{$\begin{array}{l}\text { No. of markers shared per } \\
\text { pair of RIL population in } \\
\text { consensus map }\end{array}$} \\
\hline & & & & & & & PR-02 & PR-07 & PR-15 & PR-19 \\
\hline PR-02 Orb $\times$ CDC Striker ${ }^{b}$ & 90 & 340 & 1,029 & 7 & 160 & 308 & & & & \\
\hline Pop-9 Cameor $\times$ China $^{c}$ & 124 & $405\left(499^{\mathrm{a}}\right)$ & 992 & 42 & 97 & $391\left(485^{\mathrm{a}}\right)$ & 138 & 103 & 125 & 109 \\
\hline $\begin{array}{l}\text { PR-19 Alfetta } \times \text { P651 } \\
\quad(P . \text { fulvum })^{\mathrm{d}}\end{array}$ & 144 & 940 & 457 & 49 & 90 & 303 & 105 & 57 & 88 & \\
\hline $\begin{array}{l}\text { PR-15 1-2347-144 × CDC } \\
\text { Meadow }^{\mathrm{e}}\end{array}$ & 94 & 341 & 1,019 & 4 & 172 & 308 & 124 & 100 & & \\
\hline $\begin{array}{l}\text { PR-07 Carerra } \times \text { CDC } \\
\text { Striker }^{b}\end{array}$ & 134 & 388 & 1,043 & 22 & 83 & 245 & 119 & & & \\
\hline
\end{tabular}

${ }^{\text {a }}$ Including previously published 94 frame-work markers

b Warkentin et al. (2004); see also Suppl. Info

c Bordat et al. (2011); see also Suppl. Info

d Fondevilla et al. (2005); Jha et al. (2012); see also Suppl. Info

e Warkentin et al. (2012); see also Suppl. Info

\section{SNP reporting}

Single-nucleotide polymorphisms present in the seven accessions relative to CDC Bronco were identified using Seqman Pro (DNAStar) as described in Sharpe et al. (2013) using the CDC Bronco assembly as a reference. Individual reports for each accession were parsed into spreadsheet format for comparison using a custom Perl pipeline. Only transition and transversion SNPs were reported; indels were ignored as the nature of the pyrosequencing reduces the robustness of called indels (Barbazuk et al. 2007). The final spreadsheet report (Supplementary Table S2) indicates if the SNP is the same as the reference, the alternate allele, or if there is no sequence data at that position. All lowconfidence SNPs (represented as $<80 \%$ of aligned reads have the called SNP or $<3$ aligned reads in total with called SNP) were identified and reported as being below thresholds if found in the same position as confident SNPs.

The 454 contigs were initially mapped against the annotated Medicago and soybean genomes with BLAT (Kent 2002), followed by a secondary mapping as described in Sharpe et al. (2013) using GMAP (Wu and Watanabe 2005) with the cross-species parameter for potential gene duplicate identification. Flanking sequence length and gene annotation information (Supplementary Table S2) were extracted from the sequence mapping output via a custom Perl script.

SNP validation

A subset of 32 SNPs, identified from the 454 sequence alignments, was selected for validation in silico by comparison to individual raw 454 reads, and through the development of KASP SNP assays (KBioscience, Hoddeston, UK). The SNPs were selected to assess a variety of different levels of sequence read depth, variable allele frequency, the presence or absence of SNPs in sequence flanking targeted SNPs, and marker quality score (Illumina assay design tool (ADT) score). ADT score predicts success information, validation status, and minor allele frequencies. Single SNP validation was carried out using in-house developed KASP assays as described by Sharpe et al. (2013) on the six P. sativum cultivars used for SNP discovery.

\section{Illumina GoldenGate OPA design}

From the 8,822 target SNPs identified in 4,194 contigs and selected for potential array development, an additional filtering step was employed to remove potentially heterozygous SNPs by identifying those SNPs that showed less than $100 \%$ of the reads matching either the reference or the alternate allele (1,018 SNPs; Supplementary Table S2). These SNPs were removed to avoid potential issues with assay reliability for genotyping in targeted RILs. Sequence data for the contigs surrounding the SNPs were checked for the number of base pairs to the end of the contig, to the next SNP, or to the closest splice site. Those with less than $60 \mathrm{bp}$ in this flanking region were eliminated since they cannot be used for Illumina GoldenGate arrays (Illumina Inc., San Diego, CA, USA). All remaining candidate SNPs were submitted to Illumina for assay design and a total of 7,229 SNPs were returned with ADT rank scores that indicate the likelihood of assays working based on an ideal score of 1 and acceptable to utilize with a minimum score of 0.4 ; preferential selection was given to ones scoring 
above 0.6 as recommended by Illumina. A filtered set of 3,106 SNPs, in which only the designed SNP marker with the highest ADT score per contig, was retained. Since some contigs overlapped at the same orthologous gene mapping position in Medicago, a filtered set of 2,646 SNPs, where such position duplicates had been removed, was selected. From these, all SNPs associated with a GoldenGate assay ADT score below 0.4 were removed leaving a total of 2,594 SNPs for final selection based upon observed allelic variation amongst the six $P$. sativum cultivars and a minimal amount of missing data. In total 1,245 SNPs showed a polymorphism among the cultivars, but 138 of these had large amounts of missing data leaving 1,107 selected for the GoldenGate Oligo Pool Assay (OPA) synthesis. From the remaining 1,349 SNPs that did not show any differences among the cultivars, but were polymorphic between either one or both of the P. sativum ssp. abysinicum and P. fulvum accessions and the reference CDC Bronco, a set of additional markers was chosen. Starting with missing data from just one genotype, additional markers were chosen that had the highest ADT score and a reasonably low amount of missing data (up to four genotypes with missing data). This resulted in 429 additional markers providing a final set of 1,536 SNPs represented on the pea GoldenGate array (Ps1536 OPA; Supplementary Table S2).

\section{SNP genotyping and construction of consensus pea} genetic map

A total of 586 RILs from five mapping populations (Table 2; Supplementary Table S3) were genotyped using the Ps1536 OPA using a standard GoldenGate protocol following the instructions provided by the supplier (http:// www.illumina.com/technology/goldengate_genotyping assay.ilmn). The PCR products generated were scanned for genotying using an Illumina HiScan (Illumina Inc., San Diego, CA, USA). GenomeStudio software 2010.3 (Illumina Inc., San Diego, CA, USA) was used for data clustering and allele calls were visually inspected for errors in automatic allele calling and corrected where deemed necessary. Any calls that were not clearly one allele or the other were reported as missing data to avoid errors. The segregation data in individual RIL populations were subjected to Chi square test to determine deviations from balanced segregation ratios. The markers showing Chi square values more than five were not used, except in the PR-19 RIL population where a higher degree of segregation distortion was present and Chi square values were higher.

Individual linkage maps were constructed. Maximum likelihood mapping was carried out using using JoinMap 4.0 (Van Ooijen 2006), and a minimum LOD grouping threshold of 5. Regression mapping was used to finalize the
Fig. 1 Consensus SNP linkage map of Pisum sativum generated by using five RIL mapping populations, with LG I-IV in (a) and LG $\mathrm{V}-\mathrm{VII}$ in (b). The seven linkage groups (LG I-VII) representing 7 chromosomes (given in parenthesis). Anchor markers identifying the linkage groups are shown black bold. The SNP markers common to all five RILs are shown bold red. SNP markers unique to individual RILs PR-02 (green), PR-07 (dark blue), PR-15 (brown), PR-19 (pink) and Pop-9 (light blue) are shown. Black SNP markers represent those markers shared by two or more of the RIL populations. A total of 939 loci are represented in the consensus map. The division of linkage groups into $20 \mathrm{cM}$ recombination bins is represented with blocks of different colors. Asterisk Mendel's $r$ ('rugosus' trait, where cotyledons are wrinkled with compound starch grains) is indicated on LG V (color figure online)

map order of each linkage group. The Kosambi mapping function was used to convert the recombination frequencies into $\mathrm{cM}$. The Pop9 map was constructed first and included 94 frame-work markers identified in earlier studies (Aubert et al. 2006; Deulvot et al. 2010). This allowed for the identification of individual linkage groups (LGs) and their orientation. The cross correspondence of Pop9 mapped SNP markers on individual maps was used to identify the equivalent LGs in all the RIL populations, and these were combined for map integration using the function 'join groups' in JoinMap 4.0. The consensus map output was used to generate a graphical map using MapChart 2.2 (Voorrips 2002). The consensus map was divided into $20 \mathrm{cM}$ long recombination bins to better define the position of framework markers on the consensus map. The graphical color-coded consensus pea map showing the markers contributed from each population and bin distribution is presented in Fig. 1 . The open source browser-based comparative analysis programme CMap (version 1.01; http://gmod.org/wiki/Cmap) was also utilized for the visualization of individual maps and to assess the congruency of marker positions and order among different genetic maps.

Comparative analysis of the pea genetic map with Medicago truncatula and lentil

An assessment of the syntenic relationship between pea, $M$. truncatula and lentil was undertaken using the in silico mapping data of polymorphic pea contigs mapped using BLAT (Wu and Watanabe 2005) to orthologues in the model genome together with output from a similar analysis with lentil (Sharpe et al. 2013). The comparative mapping data of macrosyntenic blocks for both pea and lentil against M. truncatula were visualized using the Circos plotting tool application (Krzywinski et al. 2009; http://circos.ca/).

Data availability

KnowPulse (http://knowpulse2.usask.ca/portal) is a repository of legume genetic and genomic data for pulse crop breeding. 
Pea LG I (2)

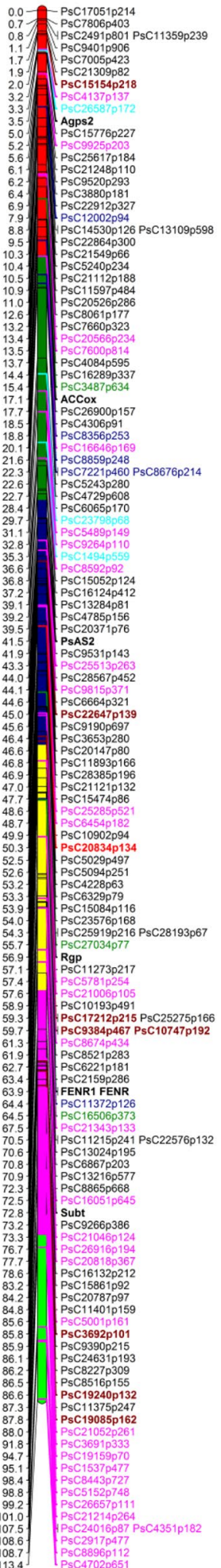

Pea LG II (6)

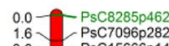

4.3 5.2 PSC185683p155

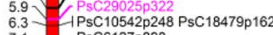

$8.1=$ PsC1 $1902 \mathrm{p} 188$

$\left.{ }_{9}^{8.9}\right)$ Gibbi

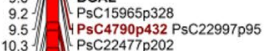

10.4

13.2 1 GCSP PsC487 4422

14.3 14.

14.8 H PsC4268p318 PsC17776p66

SC15133p661

$-P s C 5213 p 681$
$-P s C 12944062$

SC12944p62

SC12878p242

SC12039p273

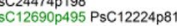

sC14928p 105

$\mathrm{PSC} 123980214$
$\mathrm{PSC} 4982083$

PsC21302p253
PsC169720253

PSC6171p84

PSC2169p461
PSC61710474

PSC17950 p474 PsC10582p78

PsC16662227

TNE003A07

PsC7665p442
PsC1764p537

PsC28442p336

-PsC2683p119

SC8994p 107 sc29120p428 PsC6113p250 PsC9109p109

28.7
28.8
29.0

PsC1648p472
PsC9819p230

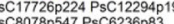

PsC6216p331
PsC12654p505

PsC6757p426

PsC7726p199
PsC5800 394

PsC20574p205
TE002011

$\mathrm{PsC2} 2677 \mathrm{p} 297$
$\mathrm{PsC} 17431 \mathrm{p} 188$

TE002D14

CWwi1

PsC6630p189

PutTip
PsDof7

PsC14311p61
PSC17639p97
PsC20372p162 PsC22916p 125

PsC149640373 PsC18956p264

PsC8833p423

PsC12544P368

- PsC7290222

PsC C 30322118
PsC16928p 164

$\mathrm{PsC16928p164}$
$\mathrm{PsC1} 12565 \mathrm{p} 223$
$\mathrm{PsC} 38230272$

$\mathrm{PSCC} 2250 \mathrm{p} 162$
$\mathrm{P} \mathrm{PSC} 7345 \mathrm{p} 63$

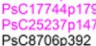

PsC8706p392
PSC25737p117

PSC14436p302
Sut1

pPgm
PsC26137p 173

PSC17102p84
PSC11711 323
PSC

PsC11711 323
PsC5597p362
PsC5013p645
PsC13131p244

PSC13131p24
PSC19573p398
PSCC17596p12
PSC1993p 347

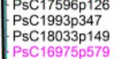

PsC5815p442
PsC15934p74

$\mathrm{PSC} 13867 \mathrm{p530}$
$\mathrm{PSC} 16861 \mathrm{p} 354$

PsC16119p474

PSC11312p220
PsC778p242
PsC9851p20

PsC1566p178

leukotr
PsC17313p181
PSC2795p765 PsC25320p116
Pea LG III (5)

0.0 - PsC9765p433

$P s C 8677$ p415
PsC53520274

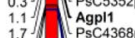

$\left.\begin{array}{l}3.3 \\ 3.4 \\ 3.6\end{array}\right) \quad\left(\begin{array}{l}\text { PsC5160p270 } \\ \text { PsC1790p38 } \\ \text { PsC11654p151 }\end{array}\right.$

$5.5 .4 P 5 C 50390842$ PsC872p28

6.3 1$)\left(\begin{array}{l}\text { PsC23493p93 } \\ \text { PsC5663p262 }\end{array}\right.$

9.1 4 PsC4599p84 PsC3395p 104

11.0 $)$ (PsC68040417

$\left.\left.\begin{array}{l}11.7 \\ 14.4\end{array}\right]\right)\left(\begin{array}{l}P s C 7893 p 98 \\ P s C 15940 \mathrm{p} 208 \\ P s C 14740177\end{array}\right.$

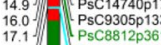

17.9
19.2
21.9

28.8 PsC14186p10

29.0 ] Clp UN

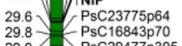

29.9 A P $29477 \mathrm{p} 305$

8.

31.2 1 (PSC17013ps

PsC26152p248
PsC18669p91 PsC26710p373

1] $\mathrm{PsC} 2298 \mathrm{p} 359$

PSC7991p378

PsC7396p78
PsC7153p118

$\mathrm{PSC} 19244 \mathrm{p} 283$
$\mathrm{PSC} 4281 \mathrm{p} 586$

PsC21413p132
PsC87220266

PsC19105p141
PsC17261p310

$\mathrm{PSC} 25874 \mathrm{p} 265$
$\mathrm{PSC} 17621 \mathrm{p} 214$

PsC28483p14

DiPeptlV
PsC11929p18
PsC527207

PSC11929p181
PSC57270267
PSC 2017907 PSC173710246

PsC29199p6

3
$\mathrm{PSC} 7922 \mathrm{p} 456$
$\mathrm{PsC} 14508 \mathrm{p} 450$

6 . PsC7220p181

F- $\begin{aligned} & \mathrm{PsC} 13905 \mathrm{p} 115 \\ & \mathrm{PsC} 13164 \mathrm{p} 99\end{aligned}$

- $\begin{aligned} & \mathrm{PsC} 3195 \mathrm{p} 368 \\ & \mathrm{PsC} 17538 \mathrm{p} 106\end{aligned}$

Agps

- $\mathrm{PsC271300229}$

$\left.\begin{array}{l}61.5 \\ 62.5\end{array}\right]-\left[\begin{array}{l}\text { PsC178092425 } \\ \text { P TE002C07 Pip1 }\end{array}\right.$

62.6
62.8
63.0

\begin{tabular}{l}
63.1 \\
63.8 \\
64.7 \\
64.8 \\
\hline
\end{tabular}

PsC25590727 72 PsC4623p 176

PSC27031p231

|l $\begin{aligned} & \text { PSC14749p226 } \\ & \text { PsC16950p61 PsC13141 220 }\end{aligned}$

ل 12.9 PsC18864p85

$79.1=\left[\begin{array}{l}73.0 \\ \text { PsC } 19393 \mathrm{p} 70 \\ \mathrm{PsC} 4478 \mathrm{p} 236\end{array}\right.$

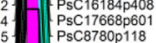

PsC2952p440

Gpt

PSC23460125

$\mathrm{PSC} 28700236$
$\mathrm{PsC} 23317 \mathrm{p} 284$

PsC3613p449

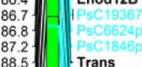

$\begin{aligned} & \text { 91.8 } \\ & 93.3 \\ & 94.7\end{aligned} \mid \begin{aligned} & \text { Trans } \\ & \text { PsC21783p63 } \\ & \text { PsC4941p186 } \\ & \text { PepC }\end{aligned}$

PepC

$\mathrm{PsC} 660505$
$\mathrm{PsC5} 4040543$

99.517 PSC5404p543

102.2
102.8

$\left.\begin{array}{l}110.0 \\ 111.5\end{array}\right]$ 盾

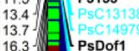

116.3
PsDof1
PsC16.4

PsC6844p132
$\mathrm{PsC26920398}$

$P \mathrm{PSC8016073}$
$\mathrm{PSC} 14587 \mathrm{p} 248$

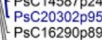

$\left(\begin{array}{l}\mathrm{PSC} 18618 \mathrm{pp78} \\ \mathrm{PsC} 14645 \mathrm{p} 416\end{array}\right.$

$\int_{\text {TE002G22 }}^{\text {PC1602 }}$

PsC23580p84
Pea LG IV (4)

0.5 - PsC8901p1

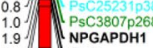

$\left.\begin{array}{l}2.6 \\ 5.1\end{array}\right]=\left[\begin{array}{l}\text { Gp22 } \\ \text { GPC21835p265 } \\ \text { PsC9619p20 }\end{array}\right.$

5.9 - PsC 8649p435

7.2
8.2

.9.

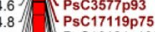

15.5 PsC19271p347

15.6.

18.6 1 H PsC3200p191 PsC17513p70

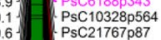

1. $\mathrm{PsC16260180}$

IPsC1965 208 PsC29043p 18

- Ugfgt

PsC7073p356

5. SPC 20003 140

PSC6766p368
PSC94187240
PSC 24877328

PSC2487p328
PSC14728 116

272 .

- Xyft

- PsC18299p388

7 - PsC7590p89

$\mathrm{PsC} 8704 \mathrm{p} 377$
$\mathrm{PsC20799p} 153$

IPsC17809p63 PsC18065p76

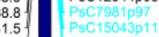

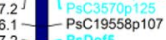

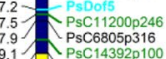

$59.9-1$ PSC7884p449

${ }_{62.0}^{61.8}$ ד $_{\mathrm{PSC} 149330313}^{\mathrm{PSC} 2663 \mathrm{p} 92}$

3.7.

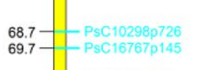

72.0

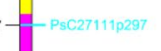

103.0 103.4 C PsC11690p23

104.2
104.7
104.7

105.9.

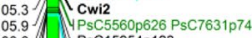

106.8 M PsC15954p193

106.9 1 106.8

$110.2]$ PsCB418223

$\begin{aligned} & 112.1 \\ & 114.7\end{aligned}-\begin{aligned} & \text { PSC 4871p } 476 \\ & \text { PsC6101p } 313\end{aligned}$

116.4- P PC6989p290

117.4 PsC1904p532

18.8 - PsC5218p76

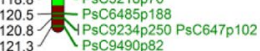



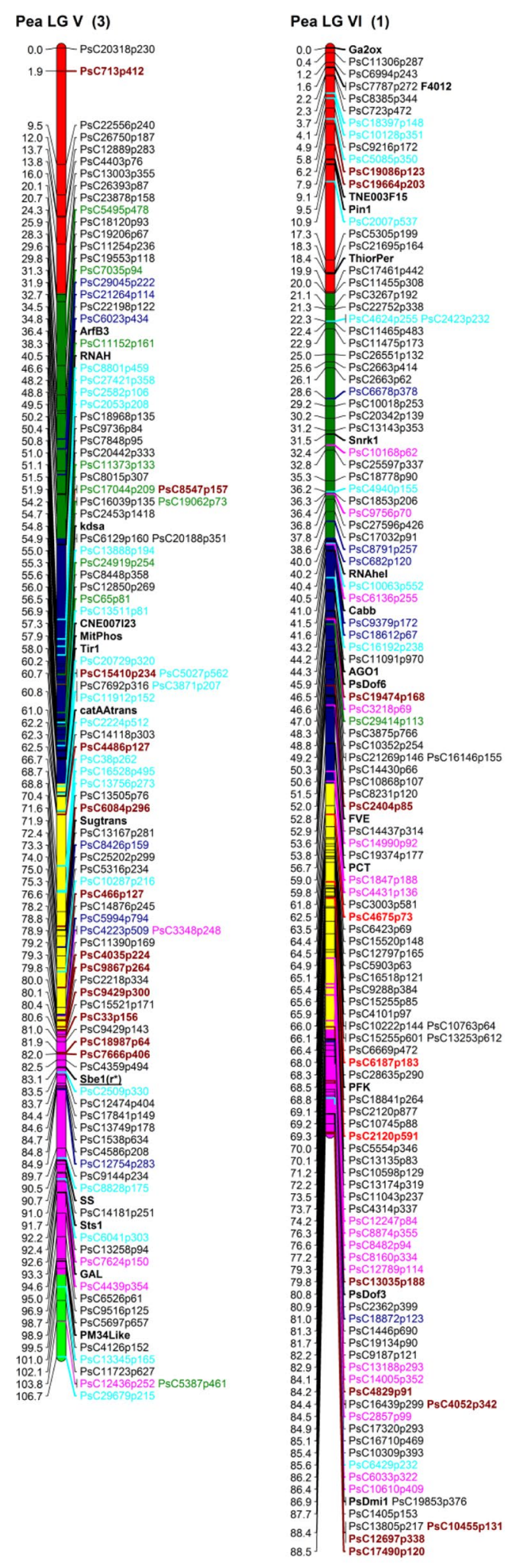

Pea LG VII (7)

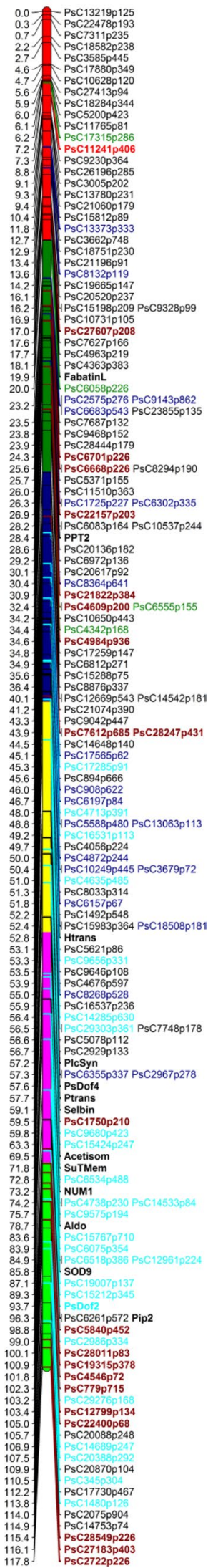

Fig. 1 continued 
The assembled 454 sequencing data for the pea reference cultivar CDC Bronco is available with all polymorphic loci and associated markers (KASP and Illumina GoldenGate arrays) indicated. Potential homologues of a gene of interest can be identified using a BLAST interface based on sequence similarity. This allows for identification of existing markers associated with the gene of interest, as well as export information on specific loci in a variety of formats to aid in marker design. A comparative GBrowse [Generic Model Organism Database (GMOD), http://gmod.org] with a Medicago genomic backbone graphically displays sequence similarity-based homology between legume species providing an alternative approach to finding candidate markers. For an overview of all data made available through this project, visit the project page (http://kn owpulse2.usask.ca/portal/node/3214575). All raw read data have been submitted to the NCBI-NIH Short Read Archive (BioProject ID: PRJNA237996; CDC Bronco (SRX555302); Alfetta (SRX555303); Nitouche SRX555304, Cooper (SRX555305), Striker (SRX555306), Orb (SRX555307), PI358610 (SRX555310) P651 (SRX555311)).

\section{Results}

454 sequencing, SNP and SRR discovery

Across all eight pea accessions, 4,008,648 sequencing reads were processed ranging from 520,797 reads in CDC Bronco to 593,701 reads in Orb (Table 1). CDC Bronco was used to generate the reference de novo assembly, resulting in a set of 29,725 high-quality reference contigs representing a significant proportion of the $3^{\prime}$ end of genes in pea. Using the custom bioinformatics pipeline (Sharpe et al. 2013), the CDC Bronco reference assembly and the $3^{\prime}$-cDNA 454 sequence data from the seven Pisum accessions, a raw total of 131,425 SNPs were identified in 20,329 contigs ( $68 \%$ of the total). Of these, $6,701(30.6 \%)$ non-redundant contigs were identified with significant homology to segments of the sequenced genomes of Medicago or soybean. Of this subset, 4,066 $(60.7 \%)$ had significant hits to annotated genes in Medicago, 1,048 (15.6\%) had significant hits to un-annotated regions in Medicago. Of those with no significant hit in Medicago, 1,587 (23.7 \%) had a significant hit to annotated genes within soybean. These contigs collectively represent 20,008 SNPs (Table 1). Information relating to the orthologous genes in the model is important since it enables an inference of the genomic position of contigs within the $P$. sativum genome, and thus aids in the selection of an optimal set of evenly distributed SNP markers. The results of the in silico mapping of polymorphic contigs to $M$. truncatula homologues are provided in Supplementary Fig. 1.
Of the 20,008 SNPs, a set of 8,822 in 4,194 contigs could be utilized for GoldenGate SNP assay development based upon there being no other observable nucleotide variation flanking the target SNPs to compromise assay performance. This set of non-redundant and high-quality SNPs comprised of 5,667 transitions (64\%) and 3,079 transversions (36\%) with 76 SNPs that could not be classified due to ambiguity of bases in the reference. An average SNP frequency of 1 SNP per $667 \mathrm{bp}$ in $P$. sativum cultivars was observed, while an average frequency of 1 SNP per 99 bp was observed when the P. fulvum and P. sativum spp. abyssinicum accessions were included. The average minor allele frequency for all SNP loci was 0.3 when all accessions were considered and 0.19 for the P. sativum cultivars alone. Five (16\%) of the 32 SNPs tested for validation failed across all genotypes (Supplementary Table S4). The other 27 SNPs were successfully separated by KASP assay, with $97 \%$ successfully matching SNP calls with the 454 sequence data in each genotype. Three of the loci with errors were only incorrect in Nitouche and Orb, suggesting they could be heterozygous at these loci. Seven genotypes had no results in the 454 data, so represent new genotypic information for these cultivars. There were five instances of the KASP assays revealing the heterozygous nature of the cultivars, not seen in the 454 sequencing data which was derived from DNA extracted from a single plant. Details regarding the parameters for the selection of SNPs for the final pea GoldenGate array (Ps1536 OPA) are provided in the "Methods".

An in silico analysis of a non-redundant set of 29,725 CDC Bronco contigs for the presence of microsatellite repeats revealed $406(1.4 \%)$ contigs that contained such a repeat with $64(16 \%)$ of these with a potential polymorphism based upon the available sequence data for all genotypes (Supplementary Table S5).

\section{SNP genotyping and genetic mapping}

Five pea RIL populations (Table 2) were genotyped with the Ps1536 GoldenGate OPA. Based upon genotype scores in the parental lines alone, a wide range of polymorphic loci was identified between 340 (22\%; PR-02) and 940 (61 \%; PR-19) loci (Table 2; Supplementary Table S3). Between 457 (30\%; PR-19) and 1,043 (68\%; PR-07) of the genotype calls were monomorphic in any one parental comparison and only a few dominant alleles were observed (ranging from 7 in PR-02 to 49 in PR-19). Six to nine percent of assays failed or had results that were unscorable in any one of the five populations, thus falling within Illumina specifications for expected success rate for new GoldenGate assays (90\% assay success). In total, 1,009 (66 \%) SNPs out of 1,536 on the array were polymorphic in at least one RIL population and could be included in linkage analysis. 
Single-nucleotide polymorphism loci exhibiting a distorted segregation with parental allele frequency of between 0.1 and 0.3 were identified in all populations but mainly in PR-19 (1 from PR-02, 1 from PR-07, 3 from PR-15, 90 from PR-19 and 9 from Pop9). However, the majority $(90 \%)$ of these distorted loci exhibited a balanced frequency greater than 0.3 in at least one of the other populations. All but 24 SNP markers from PR-19 with allele frequencies between 0.1 and 0.3 revealed a more balanced frequency in at least one of the other RIL populations. A total of $535(57 \%)$ loci identified as polymorphic in the parents of the PR-19 population (a cultivar $x$ wild accession population) exhibited extreme segregation distortion with an allele frequency below 0.1 and these loci were not included for further analysis.

The pair-wise comparisons of SNP markers shared by RIL populations are summarized in Table 2. The number of shared SNPs ranged from 57 between PR-07 and PR-19 to 138 between PR-02 and Pop9. A total of 527 (34\%) of the SNP markers on the Ps1536 array did not produce segregation data suitable for linkage analysis in any of the five populations. Of these, 348 were polymorphic amongst parental lines but exhibited extreme distortion in different RIL populations, 134 were monomorphic across all populations, and 45 produced unscorable genotyping reactions or failed across all populations.

The size of the individual maps of the five mapping populations ranged from 358.02 to $691.89 \mathrm{cM}$ with the total of mapped markers between 323 (PR-02) and 492 (Pop9). The Pop9 map also had 94 framework markers and the maximum marker interval distance was $13.51 \mathrm{cM}$. The analysis allowed the placement of 939 of the 1,009 polymorphic SNP loci to unique positions on the seven linkage groups (PsLGs) of the consensus map covering $771.6 \mathrm{cM}$ (Fig. 1). Each population contributed unique markers covering all seven LGs. The unique markers contributed by individual RIL populations to the final consensus map are color-coded in Fig. 1. While the consensus map condensed to the expected seven LGs, the individual maps ranged between 8 LG (PR-19) and 21 LGs (PR-15) (Supplementary Table S3). The consensus map was divided into 42 recombination bins, $20 \mathrm{cM}$ long. Out of these, 9 bins did not have any framework marker, while 33 had at least one framework marker with 1 bin having a maximum of seven framework markers. The number of markers present in each bin ranged from 4 to 63 markers (Supplementary Table S6). To better assess and confirm the congruency of marker positions and order among different genetic maps, a comparative analysis using CMap was carried out for the four RILs derived from cultivated parents (Fig. 2; Supplementary Fig. 2). Although overall congruence and order was good, the possibility of rearrangements on some LGs relative to different RILs was identified, perhaps most clearly at the top of PsLGVI among Pop9, PR-02 and PR-07 RILs.

Examination of the contribution of loci to the consensus map from the individual RIL populations identified groups of loci that were unique or enriched in particular populations (Fig. 1). For example, 14 markers on the end of PsLGI were only polymorphic in PR-19 while similar groups of loci are also evident on PsLGII (two blocks in PR-19; 14 markers in one and 11 in a second block), the end of PsLGIV (13 markers in PR-02) and the middle of PsLGVII (10 markers in PR-07). The 13 clustered markers mapped to PsLGIV in PR-02 had been expected to be monomorphic based on the parental genotypes from the original $3^{\prime}$-cDNA 454 sequence data.

Synteny between $P$. sativum, M. truncatula and $L$. culinaris

Comparative mapping of pea relative to both Medicago and lentil revealed large segments of shared synteny with both species (Fig. 3; Supplementary Fig. 3). PsLGI, PsLGII, PsLGIII, PsLGIV, PsLGV, and PsLGVII were relatively collinear along their entire length with individual Medicago chromosomes 5, 1, 3, 8, 7, and 4, respectively (Table 3). Pea PsLGVI exhibited shared synteny with segments of both chromosomes 2 and 6, while no other pea LG exhibited strong homology with these two chromosomes. Based on the observed syntenic relationship between the lentil LGs and the Medicago chromosomes (Sharpe et al. 2013), it was possible to infer large regions of shared synteny between pea and lentil (Fig. 3; Supplementary Fig. 3; Table 3) although in some regions the synteny is more limited (Supplementary Fig. 3).

\section{Discussion}

Genetic mapping is a pre-requisite for applications of marker-assisted selection and map-based cloning and significant efforts are being made in many crop species to develop saturated molecular maps. Despite significant advances made in the development and adoption of highthroughput genotyping and mapping tools, progress in the development of saturated molecular maps has been slow. The majority of genetic linkage maps now available have been developed by groups working independently on a wide range of mapping populations. Multiple linkage maps developed from different mapping populations recently have been aligned to generate consensus linkage maps by using common markers as framework markers. These markers identify linkage groups and define regions and orientation of the maps (Isobe et al. 2009; Gustafson et al. 2009). The use of multiple mapping populations has helped in identifying additional polymorphic markers (Studer et al. 
Fig. 2 A comparative alignment of LG3 in four RIL populations (right to left; PR-07, PR-02, Pop-9 and PR-15 using CMap version 1.01. Common markers between groups are highlighted in red to visually represent synteny of marker orders and marker positions (color figure online)

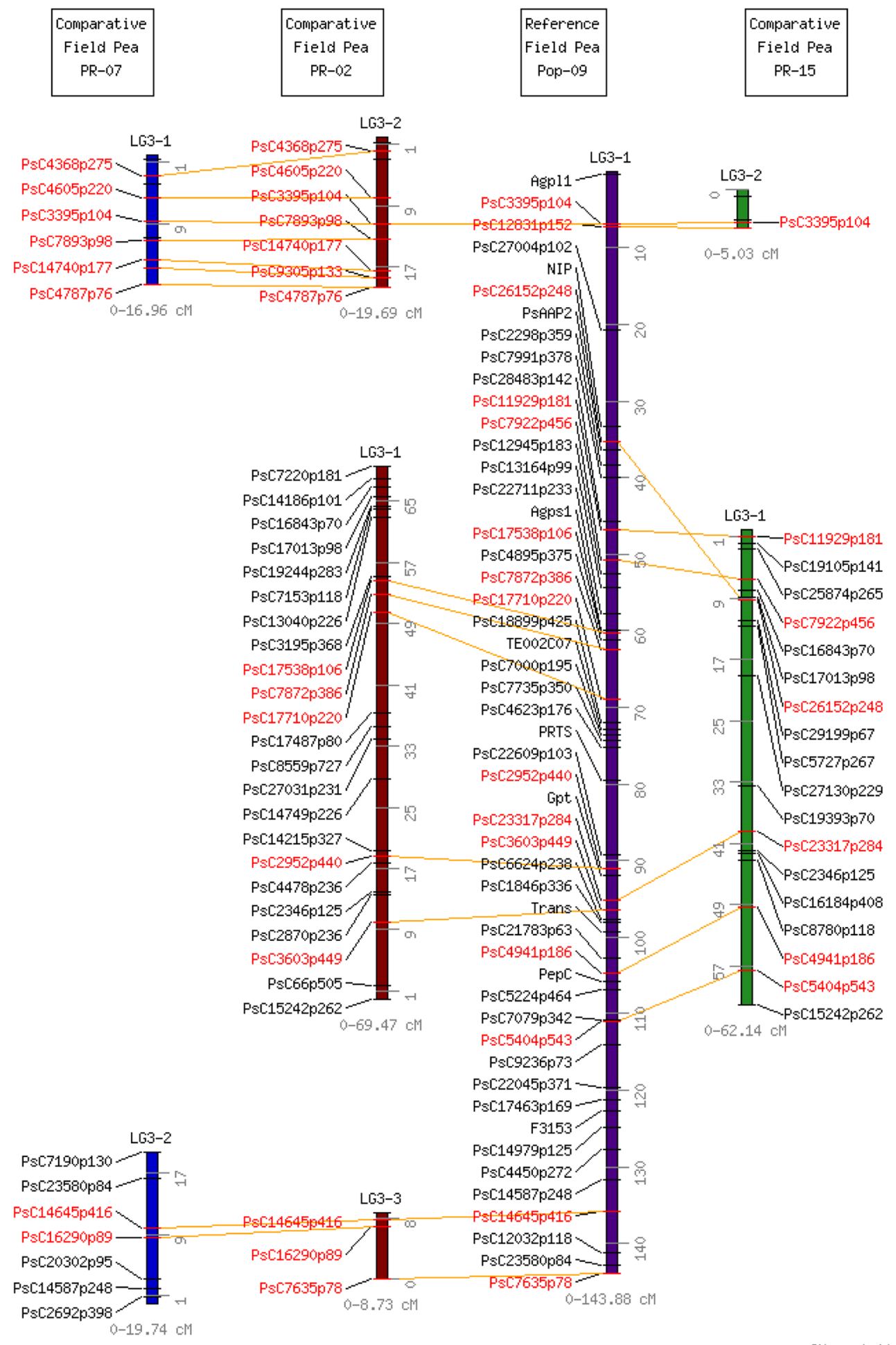

2010; Gautami et al. 2012). In this study we used 94 previously published framework markers with known locations on linkage groups to generate a consensus linkage map from five RIL populations in pea. These markers are more or less evenly distributed over all the linkage groups with an average of 12 framework markers per linkage group. A minimum of three framework markers per linkage group is suggested to be sufficient for determining orientation of the map (Gautami et al. 2012). The development of consensus maps from independent maps generated by multiple mapping populations using JoinMap software may result in discrepancies in linear order of markers due to combining 
Fig. 3 Syntenic relationships visualized by the Circos viewer (Krzywinski et al. 2009) showing extensive synteny of pea and lentil (Sharpe et al. 2013) linkage groups with the $M$. truncatula pseudochromosomes (Young et al. 2011). Ps = Pisum sativum (pea), Lc $=$ Lens culinaris (lentil), and $\mathrm{Mt}=$ Medicago truncatula

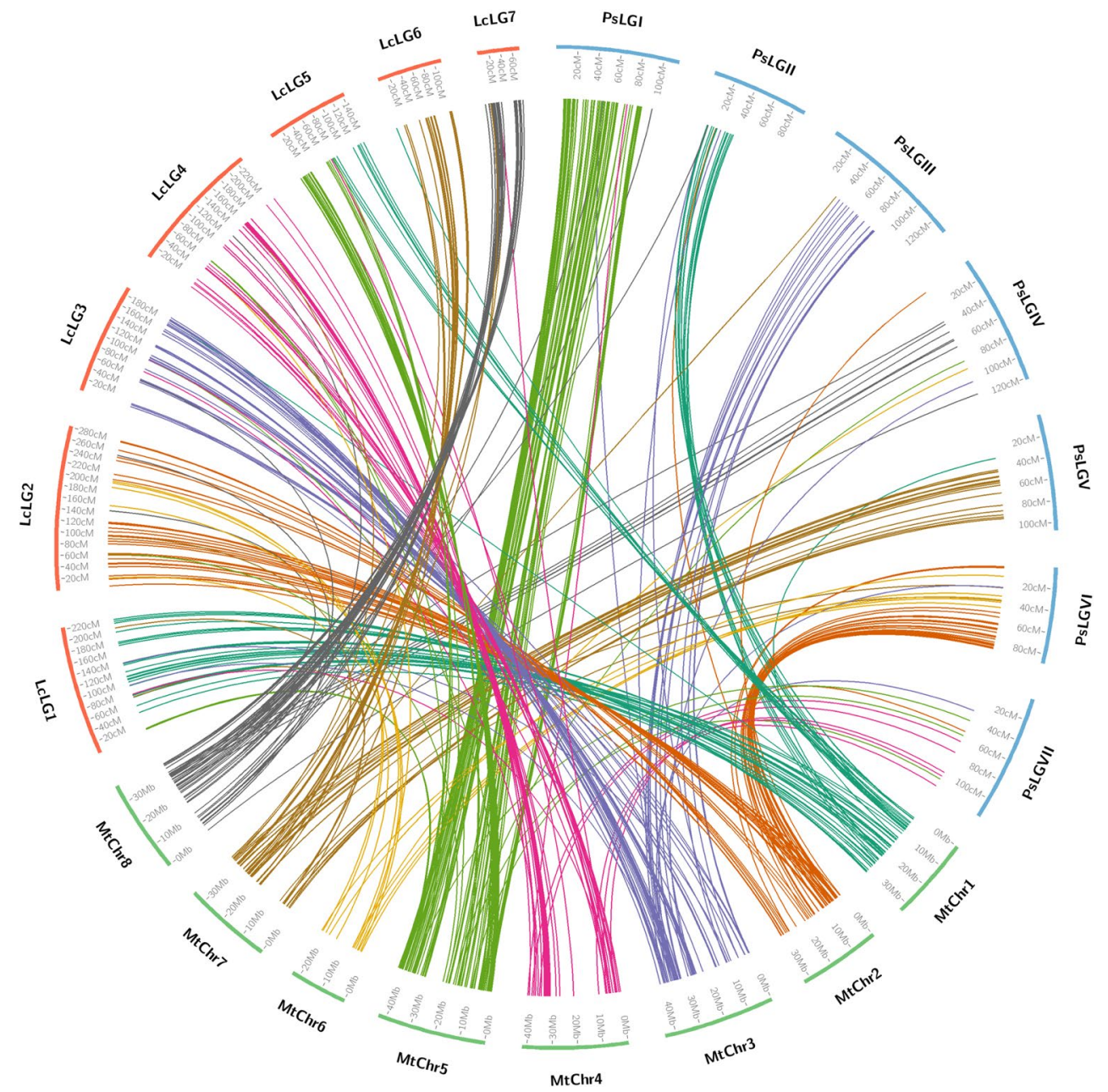

Table 3 Correspondence among pea linkage groups and $M$. truncatula pseudochromosomes and lentil linkage groups

\begin{tabular}{lll}
\hline P. sativum & M. truncatula & L. culinaris \\
\hline I & 5 & 5 \\
II & 1 & 1,5 \\
III & 3 & 3 \\
IV & 8 & 7 \\
V & 7 & 6 \\
VI & 2,6 & 2 \\
VII & 4 & 4 \\
\hline
\end{tabular}

of data from mapping populations with different recombination frequencies, genetic background, size of population, and marker density (Feltus et al. 2006). For this reason comparative mapping using CMap software was conducted to more efficiently visualize marker order in four pea RILs; an approach that aided in assessing and confirming the overall congruency of marker positions and order but also hinting at the possibility of some rearrangements on some LGs.
In our study, the markers showed varied segregation distortion in different RIL populations with PR-19 exhibiting the highest segregation distortion. Such variation in segregation distortion in individual mapping populations and inconsistencies in linear marker order in individual population maps and consensus maps have been reported previously (Gautami et al. 2012). To address these issues, several studies have reported the use of framework markers with known positions to divide linkage groups into segments representing bins which hold defined locations on chromosomes. Introduction of bins in linkage groups have been used in many crop species where saturated linkage maps are still not available (Gardiner et al. 1993; Kleinhofs and Graner 2001; Studer et al. 2010; Gautami et al. 2012). These bins can then define a fixed location on chromosomes having a framework marker. In this study, we divided the pea linkage groups into $20 \mathrm{cM}$ long bins. Out of 42 bins, the majority had an anchored framework marker identifying their fixed position on linkage groups. Introduction of bins with framework markers will help the addition of more markers in defined regions and further develop the consensus map. 
The same custom strategy for SNP discovery using 454 sequencing technology as described for lentil by Sharpe et al. (2013) was deployed in this study for the identification of a large number of SNPs from a diverse set of six pea cultivars, a wild accession of $P$. fulvum and a $P$. sativum subspp. abyssinicum land race. The $3^{\prime}$-cDNA profiling approach employed provided a robust data set with deep coverage of the targeted $3^{\prime}$ ends of genes for each of the eight genotypes. The deep coverage is significant since it provides the ability to derive both a robust reference de novo assembly for a large proportion of expressed transcripts from the harvested tissues, as well as increasing the probability of identifying highly confident SNPs for each of the genotypes. Indeed it was possible to identify a total of 29,725 non-redundant reference contigs for the CDC Bronco cultivar. This number of contigs represents a significant proportion of the expressed genes based on the fact that the diploid pea genome likely has similar gene content to the closely related model Medicago genome (45,888 protein coding transcripts; www.phytozome.net). Unfortunately, it is not possible to establish if particular transcript contigs are associated with particular tissues since the methodology utilized did not provide an efficient format for sample indexing, however, it is possible, via comparative sequence analysis, to infer both the gene function and expression characteristics from the model legume genomes, such as Medicago (Young et al. 2011) or soybean (Schmutz et al. 2010).

The identification of SNPs from the different $P$. sativum and $P$. fulvum accessions also employed the same bioinformatics pipeline previously employed in lentil (Sharpe et al. 2013) and enabled the identification of a raw total of 131,424 SNPs across 20,328 (68 \%) of the reference contigs. The level of nucleotide diversity within the P. sativum genotypes appears to follow the adapted origin of the genotypes where lower levels are observed between genotypes adapted to a North American temperate climate, and higher levels are observed between these genotypes and the $P$. sativum ssp. abyssinicum genotype (PI 358610) or the wild $P$. fulvum genotype (P651). The two wild accessions were included to broaden the genetic base of the germplasm being evaluated and thus to increase the chances for identifying SNPs. Pisum sativum subspp. abyssinicum is thought to have arisen in an independent domestication from Pisum sativum, as has Pisum fulvum. These initial SNPs were filtered to a smaller high-quality set of SNPs based upon read coverage/depth and low levels of ambiguity $(<3$ reads or $<80 \%$ concordance), as well as identified significant homology to either the model Medicago or soybean genomes. This identified a set of 20,008 high-quality SNPs across 6,707 annotated contigs. The significant decrease in the number of high-quality SNPs and contigs likely indicates two things; (1) even though the methodology enables targeted profiling of discrete regions of genes, its effect is diluted because of the presence of a large number of transcripts from a relatively small number of genes in any given tissue type; and (2) the $3^{\prime}$ end of genes can contain large stretches of untranslated regions (UTR) that can have poor levels of sequence homology even with quite closely related species. The set of SNPs selected for SNP assay development $(8,822$ SNPs in 4,194 contigs) exhibited ratios of transition SNPs (64 \%) to transversion SNPs (36\%) and a SNP frequency in P. sativum cultivars (1 SNP per $667 \mathrm{bp}$ ) that closely reflects the types of nucleotide conversions and frequency seen in similar transcript studies in pea using 454 sequencing (Kaur et al. 2012; Leonforte et al. 2013), other pulse crops such as lentil (Sharpe et al. 2013), as well as other plants (Soltis and Soltis 1998). A small number (406 (1.6\%)) of the CDC Bronco reference contigs were also found to contain a range of different microsatellite repeats, and an in silico analysis of the equivalent repeats in the sequence data from the other genotypes identified putatively polymorphic repeats. These microsatellite loci represent a resource for potential genetic marker development and complement identified repeats from other initiatives using similar sequencing strategies in this crop (Loridon et al. 2005; Kaur et al. 2012).

To select an optimal set of SNPs for representation on the Illumina GoldenGate 1,536 SNP array, the 8,822 highquality SNPs identified were screened for the removal of those SNPs that were potentially heterozygous in nature based upon the available sequence data. A total of 1,018 SNPs were identified as such indicating the residual heterozygosity in the genotypes that were selected for SNP discovery. This level of heterozygosity is not unexpected given the nature of the breeding approaches used to develop the pea cultivars in this research, i.e., none were derived from doubled haploidy. These SNPs, while potentially useful polymorphisms, were not selected for SNP assay development because we could not be confident they would be present in the targeted RILs. Of the remaining SNPs that were submitted to Illumina for assay design, it was possible to identify a set of 3,106 SNPs that best represent single contigs. This was based upon selecting SNPs with highest ADT score in cases where multiple SNPs were present in one contig. This strategy performed very well when implemented for the design of an equivalent GoldenGate 1,536 SNP array in lentil (Sharpe et al. 2013). As was the case for the lentil array, further filtering of the SNPs based upon the presence of variation amongst only the cultivated lines, the removal of lower ADT assay scores $(<0.4)$ and the removal of SNPs only identified in a limited number of lines due to limited amounts of available sequence data enabled the selection of a core set of optimal SNPs to be represented on the array $(1,107 \mathrm{SNPs})$. It should be noted that one difference from the lentil effort described by Sharpe et al. (2013), 
was that a more robust methodology for the identification of identical positional SNPs in duplicate overlapping contigs was employed in this effort; multiple approaches to map either contigs or individual reads to reference Medicago gene models were undertaken using both BLAT and GMAP. This was carried out in an effort to avoid the possibility of designed assays amplifying multiple loci within the pea genome. The supplementation of the selected 1,107 SNPs for representation on the array with an additional set of 429 markers only polymorphic between the P. fulvum and P. sativum ssp. abyssinicum genotypes and CDC Bronco completed the 1,536 SNP array. The representation of these SNPs on the array provides an enhanced ability not only to assess diversity across a wider range of germplasm, but also to better characterize segregating progeny from $P$. sativum $\times P$. fulvum and $P$. sativum $\times P$. sativum ssp. abyssinicum crosses that are available in pea breeding programs.

Validation of the selected 1,536 SNPs was established by using a subset of 32 markers for the design of KASP markers and screening against genotypes used for SNP discovery. From this it was possible to confirm that the majority of the assays would perform as expected in the larger format. A subset of five markers (16\%) did not amplify at all, and although an amount of assay failure is to be expected a subsequent analysis of these SNPs indicated that one of them had a flanking SNP in the assay design space and two of them were closely adjacent to exon/intron boundaries within the orthologous Medicago or soybean gene models. Variation in such boundaries between closely related species is to be expected and is a limitation of an approach where transcriptome data from a genome without an established reference genome is being used for SNP discovery and marker development. The validation exercise also confirmed that a significant amount of heterogeneity exists within established cultivars since it was revealed that several markers detected alleles in the cultivars that were different from the observed polymorphism in the sequence data used for SNP discovery.

The utilization of the Ps1536 GoldenGate array for genotyping in the five pea RIL populations was successful in identifying subsets of polymorphic SNP assays within each of the populations. The large difference in levels of polymorphism among the RIL populations is to be expected based upon the diverse nature of the parental material used to develop the populations. As expected the most polymorphic population (61\% polymorphic loci) was PR-19, derived from a cross between the P. sativum cultivar Alfetta and the wild $P$. fulvum accession P651. The remaining populations each had between $22 \%$ (PR-02) and $26 \%$ (Pop9) polymorphic loci, the former being derived from a cross between two modern cultivars (Orb and CDC Striker) and the latter between a modern cultivar and a Chinese landrace
(Cameor and China). The substantially greater number of polymorphic loci in the PR-19 cross likely reflects the very large degree of nucleotide diversity that exists in genic regions between the two species (Jing et al. 2007), while the level of polymorphism observed in Pop9 reflects the more moderate levels of nucleotide diversity that exists within $P$. sativum, even when including landraces. A small number of failed or unscorable assays (6-9\%) in each population is expected based upon the observed failure rate for the GoldenGate 1,536 SNP assay format (Cunningham et al. 2008), as well as the limitation of the design process inferring exon/intron boundaries from a related model species reference genome. The small number of assays where only one parental allele was observed (i.e. dominant loci) was very small with only Pop9 and PR-19 populations revealing any significant number (approx. $3 \%$ each), again likely reflecting the quite diverse nature of these crosses.

The 1,009 (66 \%) SNP assays that produced segregation data suitable for linkage analysis reflected the desire to design an array that would provide utility for genetic mapping across a broad range of crosses. The bi-allelic nature of the SNP assay format means that in any one P. sativum cross a relatively small proportion of loci (22-26\%) are polymorphic, but collectively a much larger proportion of loci can be mapped. In theory a much higher number of loci could have been mapped in PR-19 since 940 loci were identified as polymorphic between the parental lines, however, many of these loci (57\%) exhibited extreme segregation distortion (allele frequency $<0.1$ ) that was unique to this population. Of note, the majority of these loci revealed distortion skewed toward the cultivated parent genotype. The scale of this distortion is such that it indicates there were serious factors influencing the expected Mendelian ratios in the RIL population. It is possible that significant amounts of heterozygosity existed in the P. fulvum parent of the $\mathrm{F}_{1}$ such that an alternative undetectable allele is present at many loci and which resulted in the levels of distortion we observed. It is also possible that multiple gametophytic and/or genetic factors limited the representation of one parental allele over the other allele in a segregating population and there are many such reported examples in different crops (for review see Liu et al. 2010). In this case multiple significant genetic differences, such as large chromosomal translocations and inversions that are known to exist between P. sativum and P. fulvum (Errico et al. 1991), may have caused abnormal chromosome segregation in the $F_{1}$ used to generate the population. Linkage analysis of the segregation data in the five populations produced individual genetic maps for each cross that had significantly different sizes. The size of $345.3 \mathrm{cM}$ for PR-19 is particularly small and again suggests that although the parents are very polymorphic only a portion of the genome is segregating normally in this interspecific cross. 
All of the maps individually contained eight or more independent linkage groups, following the same observation in a single population described by Leonforte et al. (2013) and indicating the limitations of achieving complete genome coverage in any one bi-parental cross with a limited number of polymorphic loci. The consensus genetic map, however, derived from the five data sets, contained a total of 939 mapped loci across seven LGs with a total genetic distance of $771.6 \mathrm{cM}$ for the genome. The utilization of framework SSR markers enabled a robust integration of the consensus map with PsLG I-VII in the Pop9 map generated previously (Bordat et al. 2011). The ability to identify groups of loci within the consensus map that were primarily derived from particular crosses also established that certain regions of the genome were only polymorphic in particular crosses, most notably PR-19 and Pop9. This is not surprising considering the diverse nature of these crosses. The significant number of the groups of loci contributed by the interspecific cross PR-19 indicates that even though the cross may have a significant issue relating to normal chromosome segregation it was still possible to use the data to enhance the breadth of the consensus genetic map. The identification of clusters of loci on LG II (between marker Gibbi and cwi1), LG III (between markers PsAAP1 and NIP), and LG IV (between markers Sucsyn and Xyft) with moderate segregation distortion in individual RIL populations was also evident, with the region on LGII confirming the previously identified distortion in the region between anchor markers Gibbi and Cwi1 on this group in Pop9 (Bordat et al. 2011).

The benefit of developing these resources in pea using transcriptome data is that it is possible to take advantage of strong sequence similarity in genic regions between pea and the available sequence data from the closely related model Medicago genome (Young et al. 2011). Both pea and Medicago reside in the same galegoid clade within the papilionoid legume sub-family and are estimated to have diverged approximately 20 MYA (Cannon et al. 2009). This close relationship between the two species therefore offers the possibility of a detailed examination of the shared synteny that exists between their genomes. The availability of a similar transcriptome resource for lentil (Sharpe et al. 2013), which is another close relative within the galegoid clade, also enables a comparative analysis of synteny with both pea and Medicago.

This study identified very similar correspondence with respect to pea PsLGs, lentil LGs and Medicago chromosomes as those reported previously (Bordat et al. 2011; Smýkal et al. 2012; Leonforte et al. 2013; Sharpe et al. 2013), and previously observed rearrangements were also largely confirmed. For example PsLGI is largely collinear with Mt5, except for a small inversion of markers in the centre of the group and half of this chromosome reveals good synteny with lentil LG5. Similarly, PsLGII is largely syntenic with Mt1 with a large inversion at one end of the group while this chromosome has synteny with both lentil LG1 and LG5. Likewise PsLGIV has shared synteny with Mt8 which in turn has substantial shared synteny with lentil LG7. Interestingly, significant shared synteny between PsLGIII and Mt3 could be established with no evidence for shared synteny with Mt2 as described by Bordat et al. (2011). This lack of shared synteny could indicate technical limitations with the nature of the $3^{\prime}$ transcript profiling and resultant short contigs consisting of $3^{\prime}$ UTR sequences with little homology to the Medicago gene models, or the possibility of erroneous linkages being established between markers in the maps. It is also possible that regions of the pea genome could be more distinct because of large scale genome reorganization since the two species diverged or potentially there are specific chromosome translocations within different pea cultivars. Genome reorganization since divergence could also explain the situation in lentil where only half of Mt5 revealed substantial synteny with lentil LG5, whereas the whole chromosome is fully syntenous with PsLGI.

The genetic and genomics resources described here hold the promise to accelerate on-going efforts to improve $P$. sativum productivity and seed quality by providing a mechanism to manipulate useful variation in the crop and to analyze complex polygenic traits (e.g. QTL analysis). Together with other resources that have been recently developed (e.g., Leonforte et al. 2013), they will also assist in future efforts to develop a high-quality genome sequence for pea.

Author contributions AGS, KEB, BT, and TDW were the co-PIs on the projects that led to this manuscript; they conceived of the study, participated in its design and coordination and co-wrote the manuscript together with AS. AGS oversaw the $3^{\prime}$ cDNA library construction and sequencing portions of the work. AS, MD, RS, and KEB oversaw the genotyping and mapping portions of the work. TDW selected the germplasm used for sequencing and developed the PR-02, PR-07, PR-15, and PR-19 mapping populations together with BT, YL, ASKS, and ABJ, respectively. JB and GA developed the Pop9 mapping population. LAS prepared the data for dissemination through KnowPulse and provided bioinformatic support throughout the project. RL and JC provided input in terms of RNA extraction and established the adapted form of the $3^{\prime}$ cDNA profiling methodology. LR provided bioinformatics support from SNP selection through data analysis and prepared several figures. All authors read and approved the final manuscript.

Acknowledgments The authors thank the technical support in the National Research Council of Canada DNA Technologies Laboratory and Bioinformatics Laboratory in Saskatoon and the Saskatchewan Pulse Growers for funding associated with the Implementation of Markers for Pulses (iMAP) project. 
Conflict of interest The authors declare that they have no conflict of interest.

Ethical standards The experiments conducted in this research comply with the current laws of Canada.

Open Access This article is distributed under the terms of the Creative Commons Attribution License which permits any use, distribution, and reproduction in any medium, provided the original author(s) and the source are credited.

\section{References}

Arumuganathan K, Earle ED (1991) Nuclear DNA content of some important plant species. Mol Biol Rep 9:208-221

Aubert G, Morin J, Jacquin F, Loridon K, Quillet MC, Petit A, Rameau C, Lejeune-Hénaut I, Huguet T, Burstin J (2006) Functional mapping in pea, as an aid to the candidate gene selection and for investigating synteny with the model legume Medicago truncatula. Theor Appl Genet 112:1024-1041

Barbazuk WB, Emrich SJ, Hsin CD, Li L, Schnable PS (2007) SNP discovery via 454 transcriptome sequencing. Plant J 51:910-918

Bhattacharyya MK, Smith AM, Ellis THN, Hedley C, Martin C (1990) The wrinkled seed character of pea described by Mendel is caused by a transposon-like insertion in a gene encoding starch branching enzyme. Cell 60:115-121

Blixt S (1974) The pea. In: King RC (ed) Handbook of genetics, vol 2. Plenum Press, New York, pp 181-221

Bordat A, Savois V, Nicolas M, Salse J, Chauveau A, Bourgeois M, Potier J, Houtin H, Rond C, Murat F, Marget P, Aubert G, Burstin J (2011) Translational genomics in legumes allowed placing in silico 5460 unigenes on the pea functional map and identified candidate genes in Pisum sativum L. Genes Genomics Genet 1:93-103

Brauner S, Murphy RL, Walling JG, Przyborowski J, Weeden NF (2002) STS markers for comparative mapping in legumes. J Am Soc Hortic Sci 127:616-622

Burstin J, Deniot G, Potier J, Weinachter C, Aubert G, Baranger A (2001) Microsatellite polymorphism in Pisum sativum. Plant Breed 120:311-317

Burstin J, Marget P, Huart M, Moessner A, Mangin B, Duchene C, Desprez B, Munier-Jolain N, Duc G (2007) Developmental genes have pleiotropic effects on plant morphology and source capacity, eventually impacting on seed protein content and productivity in pea. Plant Physiol 144:768-781

Cannon SB, May GD, Jackson SA (2009) Three Sequenced Legume Genomes and Many Crop Species: Rich Opportunities for Translational Genomics. Plant Physiol 151:970-977

Clemow SR, Clairmont L, Madsen LH, Guinel FC (2011) Reproducible hairy root transformation and spot-inoculation methos to study root symbioses of pea. Plant Methods 7:46-65

Cunningham JM, Sellers TA, Schildkraut JM, Fredericksen ZS, Vierkant RA, Kelemen LE, Gadre M et al (2008) Performance of amplified DNA in an Illumina GoldenGate BeadArray assay. Cancer Epid Biomark Prev 17:1781-1789

De Caire J, Coyne CJ, Brumett S, Shultz J (2011) Additional pea EST-SSR markers for comparative mapping in pea (Pisum sativum L.). Plant Breed. doi:10.1111/j.1439-0523.2011.01917.x

Deulvot C, Charrel H, Marty A, Jacquin F, Donnadieu C, LejeuneHénaut I, Burstin J, Aubert G (2010) Highly-multiplexed SNP genotyping for genetic mapping and germplasm diversity studies in pea. BMC Genomics 11:486-495

Doyle JJ, Doyle JL (1990) Isolation of plant DNA from fresh tissue. Focus 12:13-15
Duarte J, Rivière N, Baranger A, Aubert G, Burstin J, Cornet L, Lavaud C, Lejeune-Hénaut I, Martinant JP, Pichon JP, PiletNayel ML, Boutet G (2014) Transcriptome sequencing for high throughput SNP development and genetic mapping in Pea. BMC Genomics 15:126

Ellis THN (2009) Legume genetics and breeding: the example of pea. Grain Legumes 51:14-15

Ellis THN, Poyser SJ (2002) An integrated and comparative view of pea genetic and cytogenetic maps. New Phytol 153:17-25

Ellis THN, Turner L, Hellens RP, Lee D, Harker CL, Enars C, Domoney C, Davies DR (1992) Linkage maps in pea. Genetics 130:649-663

Errico A, Conicella C, Venora G (1991) Karyotype studies on Pisum fulvum and Pisum sativum, using a chromosome image analysis system. Genome 34:105-108

Eveland A, McCarty D, Koch K (2008) Transcript profiling by 3 -untranslated region sequencing resolves expression of gene families. Plant Physiol 146:32-44

FAOSTAT (2013) Food and Agriculture Organization of the United Nations. http://faostat.fao.org

Feltus FA, Hart GE, Schertz KF, Casa AM, Kresovich S, Abraham S, Klein PE, Brown PJ, Paterson AH (2006) Alignment of genetic maps and QTLs between inter and intra specific sorghum populations. Theor Appl Genet 112:1295-1305

Fondevilla S, Avila CM, Cubero JI, Rubiales D (2005) Response to Mycosphaerella pinodes in agermplasm collection of Pisum spp. Plant Breed 124:313-315

Franssen SU, Shrestha RP, Brautigam A, Bornberg-Bauer E, Weber AP (2011) Comprehensive transcriptome analysis of the highly complex Pisum sativum genome using next generation sequencing. BMC Genomics 12:227

Gardiner JM, Coe EH, Melia-Hancock S, Hoisington DA, Chao S (1993) Development of core RFLP map in maize using an immortalized $\mathrm{F}_{2}$ population. Genetics 134:917-930

Gautami B, Fonceka D, Pandey MK, Moretzsohn MC, Sujay V, Qin H, Hong Y, Faye I, Chen X, BhanuPrakash A, Shah TM, Gowda MVC, Nigam S, Liang X, Hoisington DA, Guo B, Bertioli DJ, Rami J, Varshney RK (2012) An international reference consensus genetic map with 897 marker loci based on 11 mapping populations for tetraploid groundnut (Arachis hypogaea). PLoS One 7:e41213. doi:10.1371/journal.pone.0041213

Gilpin BJ, McCallum JA, Frew TJ, Timmerman-Vaughan GM (1997) A linkage map of the pea (Pisum sativum L.) genome containing cloned sequences of known function and expressed sequence tags (ESTs). Theor Appl Genet 95:1289-1299

Gong YM, Xu SC, Mao WH, Hu QZ, Zhang GW, Ding J, Li YD (2010) Developing new SSR markers from ESTs of pea (Pisum sativum L.). J Zhejiang Univ Sci B 11:702-707

Gustafson J, Ma XF, Korzun V, Snape J (2009) A consensus map or rye integrating mapping data from five mapping populations. Theor Appl Genet 118:793-800

Hofer J, Turner L, Moreau C, Ambrose M, Isaac P, Butcher S, Weller J, Dupin A, Dalmais M, Le Signor C, Bendahmane A, Ellis N (2009) Tendril-less regulates tendril formation in pea leaves. Plant Cell 21:420-428

Isobe S, Koliker R, Hisano H, Sasamoto S, Wada T, Kilmenko I, Okumura K, Tabata S (2009) Construction of consensus linkage map for red clover (Trifolium pratense L). BMC Plant Biol 9:57

Jha AB, Warkentin TD, Gurusamy V, Tar'an B, Banniza S (2012) Identification of mycosphaerella blight resistance in wild Pisum species for use in pea breeding. Crop Sci 52:2462-2468

Jing R, Johnson R, Seres A, Kiss G, Ambrose MJ, Knox MR, Ellis TH, Flavell AJ (2007) Gene-based sequence diversity analysis of field pea (Pisum). Genetics 177:2263-2275

Kaur S, Pembleton LW, Cogan NO, Savin KW, Leonforte T, Paull J, Materne M, Forster JW (2012) Transcriptome sequencing of field 
pea and faba bean for discovery and validation of SSR genetic markers. BMC Genomics 12:265-276

Kent WJ (2002) BLAT-the BLAST-like alignment tool. Genome Res 12:656-664

Kleinhofs A, Graner A (2001) An integrated map of the barley genome. In: Phillips RL, Vasil IK (eds) DNA markers in plants. Dordecht Kulwer Academic Publishers, The Netherlands, pp 187-199

Krzywinski M et al (2009) Circos: an Information aesthetic for comparative genomics. Genome Res 19:1639-1645

Lejeune-Hénaut I, Hanocq E, Béthencourt L, Fontaine V, Delbreil B, Morin J, Petit A, Devaux R, Boilleau M, Stempniak JJ, Thomas M, Lainé AL, Foucher F, Baranger A, Burstin J, Rameau C, Giauffret C (2008) The flowering locus $\mathrm{Hr}$ colocalizes with a major QTL affecting winter frost tolerance in Pisum sativum L. Theor Appl Genet 116:1105-1116

Leonforte A, Sudheesh S, Cogan NO, Salisbury PA, Nicolas ME, Materne M, Forster JW, Kaur S (2013) SNP marker discovery, linkage map construction and identification of QTLs for enhanced salinity tolerance in field pea (Pisum sativum L.). BMC Plant Biol 13:161

Liu J, Hutchison K, Perrone-Bizzozero N, Morgan M, Sui J, Calhoun $\mathrm{V}$ (2010) Identification of genetic and epigenetic marks involved in population structure PLOS One 5:e13209. doi:10.1371/ journal.pone.0013209

Loridon K, McPhee K, Morin J, Dubreuil P, Pilet-Nayel ML, Aubert G, Rameau C, Baranger A, Coyne C, Lejeune-Hènaut I, Burstin J (2005) Microsatellite marker polymorphism and mapping in pea (Pisum sativum L.). Theor Appl Genet 111:1022-1031

Margulies M, Egholm M, Altman WE, Attiya S, Bader JS, Bemben LA, Berka J, Braverman MS, Chen YJ, Chen Z, Dewell SB, Du L, Fierro JM, Gomes XV, Godwin BC, He W, Helgesen S, Ho $\mathrm{CH}$, Irzyk GP, Jando SC, Alenquer ML, Jarvie TP, Jirage KB, Kim JB, Knight JR, Lanza JR, Leamon JH, Lefkowitz SM, Lei $\mathrm{M}$, Li J et al (2005) Genome sequencing in microfabricated highdensity picolitre reactors. Nature 437:376-380

Meglécz E, Costedoat C, Dubut V, Gilles A, Malausa T, Pech N, Martin JF (2010) QDD: a user-friendly program to select microsatellite markers and design primers from large sequencing projects. Bioinformatics 263:403-404

Mendel G (1865) Versuche uber pflanzen-hybriden. Verh. Naturforsch. Ver. Brfinn. 4:3-47

Miesel L, Fonseca B, González S, Baezayates R, Cambiazo V, Campos R, Gonzalez M, Orellana A, Retamales J, Silva H (2005) A rapid and efficient method for purifying high quality total RNA from peaches (Prunus persica) for functional genomics analyses. Biol Res 38:83-88

Parkin IA, Clarke WE, Sidebottom C, Zhang W, Robinson SJ, Links MG, Karcz S, Higgins EE, Fobert P, Sharpe AG (2010) Towards unambiguous transcript mapping in the allotetraploid Brassica napus. Genome 53:929-938

Schmutz J, Cannon SB, Schlueter J, Ma J, Mitros T, Nelson W, Hyten DL et al (2010) Genome sequence of the palaeopolyploid soybean. Nature 463:178-183

Sharpe AG, Ramsay L, Sanderson LA, Fedoruk MJ, Clarke WE, Li R, Kagale S, Vijayan P, Vandenberg A, Bett KE (2013) Ancient orphan crop joins modern era: gene-based SNP discovery and mapping in lentil. BMC Genomics 14:192-204

Smýkal P, Hýbl M, Corander J, Jarkovský J, Flavell AJ, Griga M (2008) Genetic diversity and population structure of pea (Pisum sativum L.) varieties derived from combined retrotransposon, microsatellite and morphological marker analysis. Theor Appl Genet 117:413-424
Smýkal P, Aubert G, Burstin J, Coyne C, Ellis N, Flavell A, Ford R, Macas J, Neumann P, McPhee K, Redden R, Rubiales D, Warkentin TD (2012) Pea (Pisum sativum L.) in the genomic era. Agronomy 2:74-115

Soltis PS, Soltis DE (1998) Molecular evolution of $18 \mathrm{~S}$ rDNA in angiosperms: implications for character weighting in phylogenetic analysis. In: Soltis DE, Soltis PS, Doyle JJ (eds) Molecular systematics of plants II. Kluwer Academic Publishers, Boston, pp $188-210$

Studer B, Kolliker R, Muylle H, Asp T, Frei U, Roldan-Ruiz I, Barre P, Tomaszewski C, Meally H, Barth S, Skot L, Armstead IP, Dolstra O, Lubberstedt T (2010) EST derived SSR markers used as anchor loci for the construction of consensus linkage map in ryegrass (Lolium spp). BMC Plant Biol 10:177-187

Timmerman-Vaughan GM, Frew TJ, Butler R, Murray S, Gilpin M, Falloon K, Johnston P, Lakeman MB, Russell A, Khan T (2004) Validation of quantitative trait loci for Ascochyta blight resistance in pea (Pisum sativum L.), using populations from two crosses. Theor Appl Genet 109:1620-1631

Van Ooijen JW (2006) JoinMap ${ }^{\circledR} 4$, Software for the calculation of genetic linkage maps in experimental populations. Kyazma B.V., Wageningen, The Netherlands

van Treuren R, van Hintum TJL (2009) Comparison of anonymous and targeted molecular markers for the estimation of genetic diversity in ex situ conserved Lactuca. Theor Appl Genet 119:1265-1279

Varshney RK, Graner A, Sorrells ME (2005) Genomics-assisted breeding for crop improvement. Trends Plant Sci 10:621-630

Vignal A, Milan D, SanCristobal M, Eggen A (2002) A review on SNP and other types of molecular markers and their use in animal genetics. Genet Sel Evol 34:275-305

Voorrips RE (2002) MapChart: Software for the graphical presentation of linkage maps and QTLs. J Hered 93:77-78

Wang DG, Fan J, Siao C, Berno A, Young P, Sapolsky R, Ghandour G, Perkins N, Winchester E, Spenser J, Kruglyak L, Stein L, Hsie L, Topaloglou T, Hubbell E, Robinson E, Mittmann M, Morris MS, Shen N, Kilburn D, Rioux J, Nusbaum C, Rozen S, Hudson TJ, Lipshutz R, Chee M, Lander ES (1998) Large-scale identification, mapping and genotyping of single nucleotide polymorsphisms in the human genome. Science 280:1077-1082

Warkentin TD, Vandenberg A, Banniza S, Slinkard A (2004) CDC Striker field pea. Can J Plant Sci 84:230-240

Warkentin TD, Vandenberg A, Banniza S, Slinkard A (2005) CDC Bronco field pea. Can J Plant Sci 85:649-650

Warkentin TD, Delgerjav T, Arganosa G, Rehman AU, Bett KE, Anbessa Y, Rossnagel B, Raboy V (2012) Development and characterization of low-phytate pea. Crop Sci 52:74-78

Weeden NF, Ellis THN, Timmerman-Vaughan GM, Swiecicki WK, Rozon SM, Berdnikov VA (1998) A consensus linkage map for Pisum sativum. Pisum Genet 30:1-4

Wu TD, Watanabe CK (2005) GMAP: a genomic mapping and alignment program for mRNA and EST sequences. Bioinformatics 21:1859-1875

Young ND, Debellé F, Oldroyd G, Geurts R, Cannon SB, Udvardi MK, Benedito VA, Mayer KFX, Gouzy J, Schoof H et al (2011) The Medicago genome provides insight into the evolution of rhizobial symbioses. Nature 480:520-524

Zhu YL, Song QJ, Hyten DL, Van Tassell CP, Matukumalli LK, Grimm DR, Hyatt SM, Fickus EW, Young ND, Cregan PB (2003) Single-nucleotide polymorphisms in soybean. Genetics 163:1123-1134 